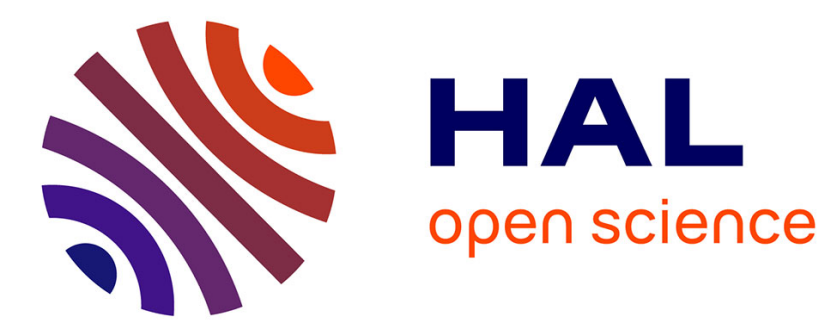

\title{
Un voyage au centre de la Terre dans Le Château des Carpathes \\ Lionel Dupuy
}

\section{To cite this version:}

Lionel Dupuy. Un voyage au centre de la Terre dans Le Château des Carpathes. Australian Journal of French Studies, 2005, pp.318-329. halshs-00476083

\section{HAL Id: halshs-00476083 \\ https://shs.hal.science/halshs-00476083}

Submitted on 23 Apr 2010

HAL is a multi-disciplinary open access archive for the deposit and dissemination of scientific research documents, whether they are published or not. The documents may come from teaching and research institutions in France or abroad, or from public or private research centers.
L'archive ouverte pluridisciplinaire HAL, est destinée au dépôt et à la diffusion de documents scientifiques de niveau recherche, publiés ou non, émanant des établissements d'enseignement et de recherche français ou étrangers, des laboratoires publics ou privés. 


\title{
Un voyage au centre de la terre dans
}

\section{Le Château des Carpathes}

\author{
LIONEL DUPUY
}

Université de Pau et des Pays de l'Adour

Le lecteur attentif de l'œuvre de Jules Verne aura souvent constaté la récurrence des mêmes thèmes, des mêmes histoires, des mêmes références. ${ }^{1}$ Or, au-delà de la simple lecture ludique et pédagogique, les romans de Jules Verne témoignent d'une formidable recherche et maturation de la part de l'auteur, autant sur le fond (la collecte d'informations et leur transmission) que sur la forme (l'articulation de ces informations dans le cadre d'une aventure plus ou moins romancée). Ses récits reposent pourtant souvent sur les mêmes bases, les mêmes structures, même si les histoires se déroulent dans des espaces géographiques très éloignés (au centre de la terre, sur terre, autour de la lune). Les périodes de temps concernées correspondent souvent à l'époque de l'auteur, mis à part bien sûr quelques cas connus (Paris an XXe siècle, pour ne citer que ce roman).

On trouve donc souvent dans les romans de Jules Verne la réitération des mêmes structures, des mêmes procédés, et parfois des mêmes idées. Tout œuvre sous-tend forcément des idées-force, et les romans de Jules Verne ne dérogent pas à la règle. L'un des tout premiers romans des Voyages Extraordinaires est Voyage au centre 
de la terre (1864). Vingt-huit ans plus tard paraîtra Le Châtean des Carpathes. ${ }^{2}$ Deux romans, deux histoires, deux époques bien différentes dans l'œuvre de Jules Verne. Pourtant il est facile de montrer comment ces deux aventures mettent en scène un même voyage dans le temps, celui au centre de la terre. Dans Voyage au centre de la terre, cela est évident, puisqu'il en constitue le titre; dans Le Château des Carpathes, un grand nombre d'analogies et de similitudes troublantes avec l'exploration souterraine permettent d'avancer aussi cette idée.

Par exemple, les volcans sont très prégnants dans les deux romans, que ce soit physiquement (Snaeffels et Stromboli dans Voyage an centre de la terre) ou toponymiquement (le col Vulkan dans Le Château des Carpathes). Ainsi, dans les deux romans, les héros sont-ils amenés à escalader une hauteur pour atteindre leur objectif. Une fois arrivés en haut, ils doivent s’introduire à l'intérieur de l'édifice (volcanique dans Voyage au centre de la terre, architectural dans Le Chatteau des Carpathes). Commence alors la descente (aux enfers). Tel Thésée perdu dans le labyrinthe, c'est grâce au fil d'Ariane qu'ils peuvent se diriger (un cours d'eau dans l'un et la voix de la Stilla dans l'autre). La même déformation du vers de Virgile renforce d'ailleurs cette dimension initiatique et mythologique, ${ }^{3}$ car, de la même sorte, dans les deux romans les héros

\footnotetext{
${ }^{1}$ Voir Volker Dehs, "Prélèvement et remploi dans l'œuvre vernienne”, Bulletin de la Société Jules Verne, 79 (1986), pp. 27-31.

${ }^{2}$ Jules Verne, Voyage au centre de la terre (Paris: Le Livre de Poche, 1996 [réédition de l'édition originale d'Hetzel, 1864]); Le Château des Carpathes (Paris: Le Livre de Poche, 1996 [réédition de l'édition originale d’Hetzel, 1892]).

${ }^{3}$ Pp. 320 et 364 dans Voyage au centre de la terre, p. 9 dans Le Château des Carpathes: "Immanis pecoris custos, immanior ipse!" (“Gardien d'un troupeau monstre, et plus monstre lui-même!').
} 
rencontrent un berger (au milieu et à la fin dans Voyage au centre de la terre, au début dans Le Château des Carpathes).

D'autre part, ces deux voyages sont fondamentalement des voyages dans le temps: dans les deux récits les héros perdent la notion du temps et sont victimes d'apparitions fantastiques (monstres des temps passés dans Voyage au centre de la terre et apparitions de personnes théoriquement mortes elles aussi dans Le Château des Carpathes). Ces apparitions semblent pourtant bien réelles aux yeux de chacun. Tout se termine finalement par une explosion (le Stromboli dans Voyage au centre de la terre, et l'explosion du château dans Le Château des Carpathes) qui permet enfin aux héros de revenir sur terre, dans le monde extérieur, normal, réel. A l’issue de ces deux voyages, les personnages principaux peuvent enfin se marier, vu qu'ils ont chacun accompli leur voyage initiatique faisant désormais d'eux de véritables hommes (Axel avec Graüben et Nic Deck avec Miriota).

Ces deux romans sont ainsi fondamentalement le récit d'aventures fantastiques mettant en scène l'imaginaire (de l'auteur et du lecteur), les sciences et techniques, et le romanesque. ${ }^{4}$ A ce titre, et au-delà de ces simples considérations et similitudes, nous pouvons déceler un seul et même voyage (dans le temps), celui au centre de la terre. La récurrence ainsi des références aux volcans et au vocabulaire qui s'y rapporte permet un premier éclairage sur cette caractéristique de l'œuvre vernienne.

Dans Voyage au centre de la terre, deux volcans bouclent le périple au centre de la terre: le Snaeffels, volcan éteint d'Islande, qui sert d'entrée, et le Stromboli, volcan actif d'Italie, plus précisément des îles éoliennes. Ce dernier sert de porte de sortie,

4 "Cette histoire n'est pas fantastique, elle n'est que romanesque" (Le Châtean des Carpathes, p. 7). 
d'ascenseur pour un retour sur terre. ${ }^{5}$ Le propre d'un volcan est de permettre la mise en communication (réelle et symbolique) de l'intérieur de la terre avec l'extérieur, donc de deux mondes totalement opposés. Au niveau mythique, l'un est du domaine de la raison, du temps linéaire, du profane, de la lumière, l'autre du domaine de l'imaginaire, du relatif, du sacrée, de l'obscurité. Deux mondes, deux univers, deux dialectiques de l'espace et du temps. C'est par la pénétration dans ce deuxième monde (le mot "pénétrer" est ici employé avec toute la symbolique sexuelle et psychanalytique qu'il requiert) que l'auteur permet d'entrer dans le domaine du fantastique, de l'irréel, du surnaturel.

Dans Le Chatean des Carpathes, la toponymie, le relief et l'architecture retenus procèdent de cette démarche: les héros font l'ascension du col Vulkan, en haut duquel se dresse un château bien mystérieux, au passé obscur, sombre (la mise en relation entre les deux mondes est évidente). Ce château a bien des allures volcaniques: son donjon principal rappelle très précisément la cheminée d'un volcan a priori éteint. ${ }^{6}$ Enfin, l'explosion finale (dans les deux romans) achève clairement cette perspective volcanique qui conduit à la destruction quasi-finale (au moins pour Le Châtean des Carpathes) de ce qui a constitué l'objet même de l'intrigue. La destruction finale de l'édifice (volcanique ou architecturale) permet de clore

\footnotetext{
${ }^{5}$ Il existe de nombreuses boucles (dialectiques) dans ce roman : ces deux volcans en forment une essentielle, et dont l'analyse approfondie permet une meilleure compréhension du roman. Voir à ce titre: Lionel Dupuy, Espace et temps dans l'cuvre de Jules Verne. Voyage au centre de la terre... et dans le temps (Dole: La Clef d'Argent, 2000). ${ }^{6}$ Voir à ce titre les nombreuses illustrations dans le roman qui renforcent cette notion.
} 
définitivement le déroulement de l'histoire, donc de mettre un terme à l'aventure proprement dite, une aventure fondamentalement initiatique.

De nombreuses références littéraires et mythologiques renforcent la dimension fantastique du Château des Carpathes et lui confèrent aussi par conséquent un caractère initiatique (autant que dans Voyage au centre de la terre). Au début de son récit, et en parlant de Frik le berger et de son troupeau, l'auteur nous dit: "L'immanum pecus paissait donc sous la conduite dudit Frik, - immanior ipse" (Le Châtean des Carpathes, p. 9). Jules Verne fait référence une fois de plus aux auteurs de l'Antiquité (comme dans ses autres romans), en reprenant ici la déformation du célèbre vers de Virgile qui - comme Simone Vierne nous le rappelle - sert de titre à un des chapitres de Notre-Dame de Paris. ${ }^{7}$ Rappelons qu'en cette fin de dix-neuvième siècle le latin et le grec sont largement étudiés dans les écoles, et notamment les vers de Virgile, ce qui explique pourquoi l'auteur n’hésite pas ainsi à faire autant de références latines, sachant pertinemment que ses lecteurs n'auront aucun mal à comprendre la référence et la pointe d'humour qui en résulte. Cette référence à Virgile par l'intermédiaire de l'œuvre de Victor Hugo permet à Jules Verne d'inscrire son roman à la fois dans une perspective mythique et moderne, notamment en se référant à l'incontournable auteur de Notre-Dame de Paris. Le caractère composite de cette citation reprise ainsi par Jules Verne est à l'image de l'œuvre de ce dernier: elle traverse le temps avec une richesse étonnante, ce que nous constatons parfaitement un siècle plus tard.

La subtilité dépend surtout du personnage auquel la citation se rapporte. En effet, nous retrouvons aussi cette citation dans Voyage au centre de la terre (p. 320). Il est

\footnotetext{
${ }^{7}$ Simone Vierne, Jules Verne. Une vie, une cuvre, une époque (Paris: Balland, 1986), p. 155. Voir la note 3 ci-dessus.
} 
intéressant de remarquer ainsi que l'emploi de cette citation, dans deux romans différents, procède donc dans les deux cas d'une caricaturisation du personnage auquel elle se rapporte (dans les deux cas il s'agit aussi d'un berger). Dans Voyage au centre de la terre, Jules Verne précise ainsi le caractère géant du berger ${ }^{8}$ tout en soulignant la monstruosité du troupeau; de même, dans Le Château des Carpathes, il est évident que le berger est plus "monstre" que géant (ou plus monstrueux que son troupeau), contrairement à son collègue vivant à quelques kilomètres sous terre. Jules Verne utilise bien les mêmes procédés littéraires pour donner la dimension fantastique et imaginaire qu'il souhaite à son récit, notamment en l'inscrivant dans la lignée des grands auteurs de l'Antiquité.

D’une part il y a donc la référence à Virgile, ce qui permet de renforcer le caractère mythique du personnage et de la situation (et par conséquent du roman, même si Jules Verne se défend dès le début de l'histoire de faire le récit d'un conte fantastique). Car rappelons que le vers de Virgile est issu des Bucoliques ("Formosi pecoris custos, formosior ipse", soit "gardien d'un beau troupeau, et plus beau luimême"), ces dernières étant constituées de dix courts dialogues de bergers. Nous pouvons ainsi traduire le texte de Jules Verne: "Le monstrueux troupeau paissait donc sous la conduite dudit Frik, - plus monstrueux lui-même que le troupeau". Jules Verne dresse une fois de plus une bien triste description de ce pauvre berger que finalement l'auteur ne semble pas porter dans son cœur.

D’autre part, il y a aussi la référence à Victor Hugo, et plus précisément à son personnage Quasimodo, gardien des monstres de la cathédrale et qui protégera

\footnotetext{
8 "C'était un géant, capable de commander à ces monstres" (V oyage au centre de la terre, p. 320).
} 
Esméralda. Nous pouvons voir ici aussi une autre référence, associant les monstres de la cathédrale aux moutons gardés par le berger qui, d'après la description de Jules Verne, est loin d'être beau, au contraire, il ressemblerait plutôt à un monstre, comme Quasimodo. Jules Verne peut ainsi placer son conte dans une double lignée historique, à la fois ancienne et classique (Virgile) et contemporaine (Hugo), ${ }^{9}$ et ainsi renforcer le caractère mythique et mystique de l'aventure qui va suivre.

Cette référence latine n'est donc pas anodine, bien au contraire, car placée dès le début de la narration (dans Le Château des Carpathes), elle permet déjà de situer le contexte dans lequel tout ce qui suit va se développer. Ce procédé qui consiste, par l'intermédiaire de références connues, à replacer son roman dans un cadre plus large, permet ainsi de donner encore plus de crédibilité à l'histoire, l'aventure (qui n'est que la suite logique d'une longue tradition) tout en conservant l'originalité de cette dernière. L'intention de Jules Verne n'en est que plus évidente.

Jules Verne fait d'autres références aussi, notamment lorsqu'il parle du propriétaire de l'auberge, Jonas (Le Château des Carpathes, p. 49). Or, ce nom de Jonas évoque directement le cinquième des petits prophètes d'Israël. Le livre biblique qui porte son nom n'est pas de lui: c'est une fiction didactique du cinquième siècle avant Jésus-Christ, qui raconte l'histoire d'une baleine qui avala Jonas et le rejeta vivant sur le rivage trois jours après. Or, trois jours, c'est justement le temps (à quelques heures près) que passe le Comte de Télek dans Le Château des Carpathes. Voilà une référence de plus (biblique ici), si l'on associe la baleine au château, et Jonas au Comte de Télek (qui sort vivant de son aventure). Le temps passé dans le château nous est d'ailleurs

\footnotetext{
${ }^{9}$ Jules Verne fait d'ailleurs une dernière référence à Victor Hugo à la page 83, concernant son recueil de poésies publié en 1856: "Comme dit le poète des
} 
donné dans le récit: “Or, comme le jeune Comte et Rotzko avaient quitté le village de Werst l'avant-veille, 11 juin, c'était la journée du 13 qui allait s'achever...” (Le Château des Carpathes, p. 182).

D'autre part, remarquons aussi que la Stilla apparaît comme l'incarnation parfaite de plusieurs femmes à la fois. Sorte de sirène, de mirage, elle symbolise l'archétype de la femme inaccessible, objet de tous les mythes, de tous les fantasmes. ${ }^{10}$ A ses apparitions sur scène succèdent plus tard des apparitions surnaturelles. Elle semble revenir d'entre les morts, ce qui renforce une fois de plus la dimension fantastique de cette aventure. Cette femme apparaît (ce verbe est fondamental en littérature fantastique) décidément inaccessible, confirmant ainsi que la science et la technologie ne peuvent pas tout faire. Telle l'étoile qui brille, ${ }^{11}$ elle est inaccessible mais elle indique le chemin à suivre. ${ }^{12}$

La voix de la Stilla, ainsi que ses autres manifestations, réelles ou virtuelles - vu que le Comte est victime à la fois d'une manipulation et d'apparitions plus ou moins concrètes - correspond également au fil d'Ariane pour le Comte perdu dans le labyrinthe du château. L'animal à abattre (le Minotaure) est incarné alors ici par le baron de Gortz. A ce titre, Orfanik, avec ses inventions, peut aussi être rattaché à

Contemplations, il 'respire de l'épouvante!"”

10 "La Stilla elle-même, par sa beauté sculpturale, sa voix parfaite, la blancheur de sa robe de scène ne symbolise-t-elle pas l'ange, la pureté virginale de la mythologie judéo-chrétienne?" (Agnès Perrin, "Étude d'une ouvre intégrale: Le Château des Carpathes", Nouvelle Revue Pédagogique, 5 (2000), 30).

${ }^{11}$ Stilla $=$ stella $=$ étoile. D'ailleurs, comme une étoile qui brille encore, la Stilla est bel et bien morte depuis longtemps.

${ }^{12}$ Comme pour la naissance de Jésus. Nous sommes évidemment dans la continuité des références bibliques. 
Dédale, le maître de lieux. Les coins et recoins du château symbolisent le labyrinthe dont il faut sortir vainqueur, ce qu'arrivera à faire le baron. Pour autant, il n'aura pas sauvé la Stilla: indirectement, en éclaircissant le mystère, c'est Miriota qu’il délivre en permettant à Nic Deck de pouvoir enfin se marier tranquillement avec elle. D'ailleurs, le nom de cette dernière n'évoque-t-il pas le miroir, autrement dit l'image renvoyée (et renversée) de la Stilla? Enfin, le comte de Télek peut être associé à Thésée, célèbre roi de la mythologie grecque, qui sortira vainqueur de cette aventure, grâce à l'aide d'Ariane, en suivant sa voix (ou sa voie). Dans le mythe, Ariane tombe amoureuse de Thésée. Cette liaison est symbolisée ici par le mariage (qui peut enfin avoir lieu) entre Nic Deck et Miriota. Finalement, le comte (donc Thésée) finit par sauver du mal ces pauvres habitants de Werst. Pour simplifier et résumer, nous proposons alors les associations suivantes:

Chatteau des Carpathes

$\begin{array}{lll}\text { Stilla } & = & \text { Ariane } / \text { Fil d'Ariane } \\ \text { Baron de Gortz } & =\text { Minotaure } \\ \text { Orfanik } & =\text { Dédale } \\ \text { Franz de Télek } & =\text { Thésée } \\ \text { Château des Carpathes } & =\text { Labyrinthe }\end{array}$

Voyage au centre de la terre

$\begin{array}{lll}\text { Cours d'eau } & =\text { Ariane } / \text { Fil d'Ariane } \\ \text { Lidenbrock } & =\text { Minotaure } \\ \text { Saknussemm } & =\text { Dédale } \\ \text { Axel } & =\text { Thésée } \\ \text { Centre de la terre } & =\text { Labyrinthe }\end{array}$


Pour parachever la référence, Jules Verne nous confirme définitivement le choix de ce parallèle mythologique: “Tel était l'ensemble de ce château des Carpathes, dont le plan géométral offrait un système aussi compliqué que ceux des Labyrinthes de Porsenna, de Lemnos ou de Crète. Tel que Thésée, pour conquérir la fille de Minos, c'était aussi un sentiment intense, irrésistible, qui venait d'attirer le jeune comte à travers les infinis méandres de ce burg. Y trouverait-il le fil d'Ariane qui servit à guider le héros grec?" (Le Château des Carpathes, p. 171) Ainsi, tout comme le célèbre héros, le comte s'enfonce dans le labyrinthe sans avoir aucun point de repère. Cette absence de repère et cette référence mythologique sont aussi à la base de tous les voyages initiatiques parsemés d'aventures fantastiques (comme dans les récits de la mythologie grecque et romaine). ${ }^{13}$

Enfin, et concernant le récit que fait le docteur Patak aux habitants de Werst, Jules Verne utilise une dernière référence: “Oui! Il voulait passer la nuit dans le donjon... autant dire la chambre à coucher de Belzébuth!” (Le Château des Carpathes, p. 102) Belzébuth est une divinité cananéenne, devenue chez les juifs et les chrétiens le prince des démons. Cette dernière référence (qui renforce le caractère fantastique du donjon et du château) permet une fois de plus à Jules Verne d'associer le lieu de l'action principale à un personnage fantastique mais pourtant bien ancré dans nos esprits et ceux des lecteurs. Tout cela participe donc d'une construction littéraire

\footnotetext{
${ }^{13}$ Rappelons d'autre part que c'est aussi une voix intrigante entendue dans l'auberge qui est à l'origine de cette aventure au château des Carpathes: 'La voix est le fil. C'est en suivant le filet de voix que Franz remonte jusqu'au château, s'enfonce dans les souterrains, arrive dans le puits avant d'accéder au donjon. Convoquer le fil d'Ariane, le labyrinthe et le jeu de l'oie avec ses cases obligées coule de source et justifie la
} 
parfaitement étudiée où tous les détails sont soigneusement agencés afin de mettre en place un récit fantastique, bien qu'étant à la fin complètement expliqué. Cette dimension fantastique appuyée par l'emploi de références mythologiques et littéraires introduit aussi une autre thématique, celle d'une recherche de la femme et de la féminité dans le cadre d'un processus (mythique lui-aussi): la maïeutique. Cette thématique est celle indirectement d'une autre réflexion, celle de la place de l'homme sur terre face à un environnement qu'il maîtrise plus ou moins.

Les romans de Jules Verne sont ainsi les témoignages d'une réflexion très avancée pour l'époque concernant notamment les rapports de l'homme avec son environnement, et plus particulièrement sur la thématique de "l'habiter" (ö̈kos en grec $=$ habitat). Car en effet, comment ne pas non plus déceler dans Vyage au centre de la terre et dans Le Château des Carpathes respectivement une réflexion sur des écosystèmes disparus (époques géologiques) et sur l'influence d'un site et d'une situation géographique peu favorables à la curiosité scientifique et au développement de l'esprit critique? Ces deux romans sont fondamentalement des voyages initiatiques où l'auteur s'interroge sur la place de l'homme sur terre (ou sous terre)..$^{14}$ D'ailleurs, le caractère souvent géographique des aventures narrées participe activement de cette démarche analytique: comment les gens vivent-ils ailleurs (dimension de l'espace)? comment vécurent-ils autrefois (dimension du temps)? ou enfin, comment vivent-ils

lecture de ce roman comme récit d'une initiation" (Le Château des Carpathes (Paris: Éditions Babel, 1997). Cf. l'analyse du roman par Jean-Louis Leutrat, pages 231-232). ${ }^{14}$ Lorsque que le Comte de Télek pénètre dans le château, il commence lui aussi par descendre, comme s'il devait atteindre le fond pour mieux remonter (principe même de l'initiation): "Il se baissa, et sa main sentit une marche en contrebas, puis une 
ailleurs et maintenant (dialectique de l'espace et du temps)? Ainsi, ces dimensions de l'espace et du temps se mélangent-elles parfois dans le cadre d'une dialectique qui permet la mise en place souvent d'une troisième dimension (voire parfois plus). Cette dimension nouvelle (initiatique, fantastique, imaginaire) interroge souvent le lecteur de manière inconsciente. ${ }^{15}$ C'est peut-être là aussi que se situe la richesse de l'œuvre de Jules Verne, car elle est capable de divertir tout en éduquant et interrogeant la sagacité du lecteur. Ce processus résiste à l'analyse logique, mais force est de constater que tous les romans de Jules Verne disposent d'une puissance de réflexion sur l'époque considérée indiscutable. Ces deux romans montrent clairement que la place de l'homme sur terre fait partie intégrante des préoccupations de l'auteur. Mais plus que la place de l'homme, n'est-ce pas aussi une réflexion sur la place de la femme dans la vie de l'homme? Car l'objet central du Voyage au centre de la terre est bien le centre de la terre (et aussi, indirectement, le ventre de la femme, à savoir celui de la mère) et dans Le Château des Carpathes la réflexion porte sur la place de la femme en tant que femme, qu'épouse (celle qui deviendra plus tard une mère ou qui aurait pu le devenir). Dans les deux cas la femme est physiquement absente mais symboliquement très présente. Démontrer que l'on peut retrouver un voyage au centre de la terre dans Le Château des Carpathes, c'est donc démontrer indirectement que ces deux romans mettent en scène de manière symbolique la Femme. Associer le voyage au centre de la terre avec le jeu de mots "voyage dans le ventre de la mère"

seconde. Il y avait là un escalier. Cet escalier s'enfonçait dans les fondations du château, et peut-être n'avait-il pas d'issue?" (Le Château des Carpathes, p. 173 et 174). ${ }^{15}$ Sur la dimension du temps dans Le Chateau des Carpathes, voir Patrick Avrane, "La Voix du château", in Jules Verne 7: Voir du feu (Paris: Minard, 1994), 89-99. 
est d'une évidence indiscutable. Néanmoins, cette évidence sous-tend une réalité: l'absence d'une femme pourtant omniprésente!

Lorsque le Comte de Télek pénètre dans le château des Carpathes, il pénètre dans l'antre du château, afin d'accéder à son amour éternel. Le résultat est terrible: tout cela n'est qu'illusion et manipulation. La Stilla est bel et bien morte, même une deuxième fois en l'occurrence. Cette double mort permet néanmoins deux choses: d'une part le comte de Télek peut enfin faire son deuil, d'autre part, cette renaissance permet également un retour dans le monde "normal". Finalement, Nic Deck peut se marier, tout comme Axel avec Graüben dans Voyage au centre de la terre. L'explosion du Stromboli d'un côté et du château de l'autre ne symbolise-t-elle pas un accouchement provoqué par une fécondation volontaire mais inattendue? ${ }^{16}$

Nous nous retrouvons toujours ainsi dans la même dialectique de l'espace et du temps décrite précédemment: ces différents voyages permettent finalement un retour sur soi, une sorte d'introspection qui permettra une renaissance. Si la femme est souvent absente des romans de Jules Verne, la symbolique qu'elle représente est omniprésente: la mer intérieure dans Voyage au centre de la terre, la Stilla dans Le Château des Carpathes, la mer (et son homonyme inévitable: la mère) dans bon nombre de romans, sans oublier l'île (on dit bien une île), la terre qui représente le ventre de la mère, etc. Voyager dans les entrailles de la terre ou de n'importe quelle autre structure aussi symbolique, c'est donc incontestablement voyager dans le ventre de la mère, donc procéder à une re-naissance. La dimension initiatique qui en découle est évidemment importante et incontournable: le périple et les différentes difficultés que

${ }^{16}$ Dans le même ordre d'idées, voir Michel Cardenas-Sanchez, "Voyage au centre de la mère", Réalités Pédiatriques, 76 (2002), 40-42. 
les héros ont su surmonter font d'eux des hommes désormais fondamentalement différents de ce qu'ils étaient au début du roman. Tel Axel et Nic Deck qui peuvent enfin épouser leurs fiancées, tel Lidenbrock qui est maintenant célèbre et reconnu, tel le comte de Télek qui peut enfin faire le deuil de la mort de sa femme et par conséquent commencer une nouvelle vie.

Les pérégrinations des héros dans le cadre de leurs aventures respectives (au centre de la terre ou dans le château des Carpathes) s'accompagnent donc d'apparitions fantastiques, mais bien réelles dans les romans (et aux yeux des héros). Dans les deux cas, ces voyages sont aussi des voyages dans le temps, ${ }^{17}$ des retours vers le passé, à la recherche de l'impossible et de l'inaccessible (le centre de la terre et la Stilla). Pourtant, dans les deux cas, deux personnages prétendent y être parvenus, ou du moins en donnent l'illusion: Saknussemm prétend ainsi avoir atteint le centre de la terre, comme le Baron de Gortz qui, avec ses illusions (d'optique), donne l'impression de "posséder" la Stilla, et par conséquent d’entretenir à jamais cet amour qui lui fut pourtant impossible du vivant de la cantatrice.

La fin du dix-neuvième siècle est une période charnière en matière de progrès scientifiques et techniques: les romans de Jules Verne témoignent parfaitement de la dialectique du développement des progrès scientifiques et techniques face à la survivance de nombreuses croyances et mythes issus du fond des âges. Cette cohabitation difficile, dans l'espace et dans le temps, de deux univers totalement opposés, est à la base de la mise en place des dimensions fantastique et imaginaire dans les romans de Jules Verne. Un exemple cristallise parfaitement cette 
cohabitation. Dans Voyage au centre de la terre, le professeur Lidenbrock est l'auteur d'un Traité de Cristallographie transcendante (Voyage au centre de la terre, p. 5). Ce traité, purement imaginaire évidemment, "cristallise" parfaitement le contexte dans lequel

Jules Verne rédige ces romans, et avec quelles intentions. Car si la cristallographie existe réellement, son association avec l'adjectif "transcendante" constitue bien évidemment une référence directe aux traités d'alchimie des siècles précédents, dont l'objectif principal était celui de transformer le plomb en or, notamment avec des expériences chimiques aussi surnaturelles qu'impossibles, et par conséquent forcément inefficaces. Néanmoins, il s'agit d'associer la science à l'imaginaire et au surnaturel, ce que l'on retrouve parfaitement dans le cadre du Château des Carpathes. La transcendance, au sens philosophique du terme, caractérise ce qui se situe hors d'atteinte de l'expérience et de la pensée de l'homme. Tout cela se situe à l'opposée de la science qui se base avant tout sur l'expérience, d'où une association impossible (techniquement) de la cristallographie avec cet adjectif, mais qui du point de vue de la construction du récit témoigne d'une parfaite maîtrise de la complexité du sujet traité et de son caractère atemporel. C'est aussi par de petits clins d'œil pareils que Jules Verne montre sa volonté de dépasser les disciplines dans le but de renforcer le caractère extraordinaire des voyages qu'il nous propose.

Revenons plus précisément à notre voyage au centre de la terre: les illusions d'optique mises en place par Orfanik et le Baron de Gortz transcendent littéralement l'esprit critique du Comte de Télek. Mais, finalement, dans cette histoire, le Baron de

\footnotetext{
${ }^{17}$ Voir à ce sujet William Butcher, Verne's Joumey to the Center of the Self: Space and Time in the "Voyages extraordinaires" (New York: Saint Martin's Press; London: Macmillan,
} 
Gortz se retrouve autant pris dans son piège que le Comte. Durant son périple, et de la même sorte que pour les héros de Voyage au centre de la terre, le Comte de Télek est victime d'apparitions surnaturelles, fantastiques, tels les monstres antédiluviens observés dans le centre de la terre. La perte de la notion du temps est corrélative d'un état d'esprit et d'un personnage qui n'a plus de points de repère. Ce que l'œil voit estil réel ou le fruit de l'imagination à la fois du héros (victime de la plume de Jules Verne) et du lecteur? Car si l'on voit réellement des monstres (préhistoriques dans le premier texte, surnaturels dans le deuxième), ces derniers ne sont-ils pas imaginaires, irréels, donc fantastiques, car n'appartenant pas au quotidien du personnage principal?

Le Chattean des Carpathes, comme Voyage au centre de la terre, est ainsi un voyage dans le temps (triplement même), où l'ubiquité temporelle est permise par l'écriture de Jules Verne. En effet, et comme nous l'avons précisé dans une autre étude, ces deux romans sont naturellement des voyages dans le temps: ${ }^{18}$ à la découverte des écosystèmes disparus du Voyage au centre de la terre correspond dans Le Château des Carpathes la découverte d'un monde qui vit en marge des progrès scientifiques et techniques; de la même sorte qu'à la descente dans le centre de la terre correspond la descente dans les entrailles du château; et enfin, à la rencontre des personnages morts du voyage au centre (Arne Saknussemm, l'homme du quaternaire, etc.) correspond un voyage dans le temps qui permet au comte de Télek de se croire en présence de sa femme pourtant bien morte. Trois périodes de temps différentes: le voyage en luimême (le temps ordinaire, mesuré, qui s'écoule normalement), le voyage dans les

1990).

${ }^{18}$ Dupuy, Espace et temps dans l'curre de Jules Verne. 
temps historiques (préhistoriques et géologiques dans Voyage au centre de la terre), et le voyage dans les temps psychologiques (à la perte de la notion du temps vécue aussi dans Voyage au centre de la terre correspond le voyage dans le passé et l'histoire du Comte de Télek avec sa femme). D’ailleurs l'écriture de Jules Verne reflète bien cette logique. Les voyages dans les temps psychologiques sont appuyés par l'auteur par des digressions, obligatoires, qui permettent de relater l'histoire des personnages avant de revenir à la logique du roman qui est une invitation évidente au voyage (celui du lecteur).

Tous ces voyages dans le temps procèdent évidemment d'une démarche volontaire de la part de l'auteur, car ils permettent principalement la mise en place de la dimension fantastique souhaitée par Jules Verne. Ainsi, le fait de rencontrer des morts (qu'ils soient bien morts ou étonnamment en vie) permet donc de transporter à la fois les héros mais aussi le lecteur dans un univers mystérieux mais ô combien attrayant. Jules Verne ne déclarait-il pas: "Mon but a été de dépeindre la Terre, et pas seulement la Terre, mais l'univers, car j'ai quelquefois transporté mes lecteurs loin de la Terre dans mes romans" (1893). ${ }^{19}$

Comme nous avons essayé de le montrer, et comme d'autres l'ont fait bien avant nous, l'œuvre de Jules Verne doit être lue bien au-delà des simples considérations ludiques et pédagogiques, certes présentes, mais que l'on nous présente habituellement. Car ces dernières sont trop souvent réductrices, et elles ont nui à la réputation de l'auteur en son temps et même par la suite. Peut-être cela est-il le signe du caractère précurseur de son œuvre. Quoi qu'il en soit, force est de

19 Propos rapporté par Jean-Paul Dekiss, Jules Verne. Le rêve du progrès, (Paris: Gallimard, 1996), p. 146. 
constater que les romans de Jules Verne sont d'une formidable puissance de réflexion et d'interrogations diverses, conscientes ou inconscientes. C'est à ce titre que s'inscrit notre démarche, qui entend participer à la réhabilitation d'un auteur passionnant et passionné.

Ainsi, le thème du voyage au centre de la terre peut être décelé dans le cadre du Châtean des Carpathes. Les volcans, la mythologie, la recherche de la femme (sous toutes ses formes, au sens propre comme au sens figuré) ou encore les dimensions fantastique et imaginaire sont-ils autant de supports que Jules Verne a utilisés pour construire ses aventures. Or, c'est parce que ces supports renvoient directement à des thèmes et à des sujets familiers que nous nous identifions aussi facilement à ses héros et à leurs aventures. Il y a donc bel et bien un voyage au centre de la terre dans Le Château des Carpathes.

Les romans de Jules Verne sont aussi les témoignages d'un auteur face à son époque mais avec certaines réflexions en avance d'un siècle (c'est à ce titre que nous célébrons le centenaire de sa mort cette année). Tous ces témoignages s’appuient sur des structures simples, des principes logiques (parfois quasi-mathématiques ${ }^{20}$ ) qui renvoient à des utopies propres à tous les temps et tous les hommes: découvrir le centre de la terre, inventer la machine à remonter le temps, ressusciter les morts, etc. Ces utopies motivent ainsi des voyages, pour le moins extraordinaires, tel en est d'ailleurs le titre donné au corpus composé par une bonne partie des romans de Jules Verne. La recherche du centre de la terre participe ainsi activement d'une de ces

${ }^{20}$ Le Tour du monde en 80 jours en est d'ailleurs l'archétype, notamment avec son héros principal, Phileas Fogg.Voir à ce titre: Lionel Dupuy, Itinéraire d'un voyage initiatique. Le tour du monde en 80 jours de Jules Verne (Dole: La Clef d'Argent, 2002). 
utopies, dont le talent de Jules Verne a été d'être capable de le décliner au sens propre (Voyage au centre de la terre) comme au figuré (Le Châtean des Carpathes). Les utopies motivent les voyages. N'oublions pas alors, dans cette perspective du voyage dans l'espace et dans le temps, l'une des principales dimensions du voyage, à savoir sa dimension initiatique:

Heureux qui, comme Ulysse, a fait un beau voyage,

Ou comme cestuy-là qui conquit la toison,

Et puis est retourné, plein d'usage et raison,

Vivre entre ses parents le reste de son âge! 